\title{
Effects of Ambient Temperature and Shaft Power Variations on Creep Life Consumption of Industrial Gas Turbine Blades
}

\author{
Ebigenibo Genuine Saturday', Thank-God Isaiah ${ }^{2}$ \\ ${ }^{1}$ Department of Mechanical Engineering, University of Port Harcourt, Port Harcourt, Nigeria \\ ${ }^{2}$ Department of Mechanical Engineering, Niger Delta University, Amassoma, Nigeria \\ Email: satebi@yahoo.com
}

How to cite this paper: Saturday, E.G. and Isaiah, T.-G. (2018) Effects of Ambient Temperature and Shaft Power Variations on Creep Life Consumption of Industrial Gas Turbine Blades. Energy and Power Engineering, 10, 120-131.

https://doi.org/10.4236/epe.2018.103009

Received: January 31, 2018

Accepted: March 27, 2018

Published: March 30, 2018

Copyright (c) 2018 by authors and Scientific Research Publishing Inc. This work is licensed under the Creative Commons Attribution International License (CC BY 4.0).

http://creativecommons.org/licenses/by/4.0/

\begin{abstract}
The effects of ambient temperature and shaft power variations on creep life consumption of compressor turbine blades of LM2500+ industrial gas turbine engine are investigated in this work. An engine model was created in PYTHIA (Cranfield University's gas turbine software) for the analysis. Blade thermal and stress models were developed and used together with Larson-Miller Parameter method for life analysis. Mean life reduction index, which is the propensity of a given effect to reduce engine life by half, is developed for each effect and applied in this research to compare the impacts of the different effects on the blade creep life. It was observed that blade life will be halved when ambient temperature is increased by 8.11 units while $13.64 \%$ increase in shaft power reduces blade life by a factor of 2 . The results of this work will guide engine operators in making decisions concerning operating at part loads.
\end{abstract}

\section{Keywords}

Creep Life Consumption, Engine Model, Thermal Model, Stress model, Mean Life Reduction Index

\section{Introduction}

Gas turbine hot section components such as the high pressure (HP) turbine blades are exposed to highest gas temperatures and are more likely to fail due to creep. There are several methods of investigating turbine blade life [1] [2] [3] [4]. The rate of creep consumption of materials increases with mechanical as well as thermal load [5] [6] [7] [8]. This is because the bonds holding the atoms together in the metallic structures get weak with increase in temperature and 
applied stress. Thus, the creep life of the HP turbine blades are affected dominantly by the temperature of the blades and the stresses the blades are imposed with. The temperature of the hot gases in contact with the blades is affected by the ambient temperature in which the engine operates, the shaft power level, and the degradation of the engine. The temperature of the gases of the blades is exposed to increase with the increase of ambient temperature if the engine is to keep the same power level. High engine power output leads to high temperature of the hot gases. A degraded engine will have to work harder to deliver the same power, which will lead to the increase of the hot gas temperature, but, in this work, only the effects of ambient temperature and shaft power level variations on the HP turbine blades of the LM200+ gas turbine engine are studied and presented.

The effects of various engine operating conditions and design parameters on engine life have been investigated by many researchers [2] [8] [9] but the effect of any engine operation parameter on creep life in relation to other parameters is often overlooked or loosely investigated. For instance, the effect of ambient temperature on creep life may be studied without considering the effect at different power levels. The effect that an increase in ambient temperature has on creep life will be different at different power levels. Thus, pinning the response of engine creep life to ambient temperature variation on the power level will give a general picture of how engine creep life responds to ambient temperature variation. In this research, the effects of ambient temperature variation at different power levels and power variation at different ambient temperatures on creep life are investigated.

The effect of the variation of any parameter such as ambient temperature and engine shaft power level on engine creep life consumption could be expressed in terms of creep factor of engine operation [10] instead of creep life. The creep factor is the ratio of the remaining life of the engine component at a given point of engine operation to a reference life, and this could be extended to accommodate several operating points of the engine exploiting fractional life accumulation techniques [11]. Since the creep factor is derived from creep life, any effect that leads to decrease or increase in creep life will also lead to decrease or increase in creep factor. To be able to compare the impact of the different effects on creep life, a new parameter, life reduction index, which is the propensity for the given effect to reduce the life of the engine components by half, is developed for each effect. One has no control of the ambient conditions but the power level of the engine could be adjusted for part load operation. The engine operates in several off-design conditions in practice. Knowing how engine creep life responds to ambient at different power levels, the engine operators will be able to take decision on when to operate at part load if the aim is to extend engine creep life. This is one critical issue addressed in this research.

\section{Methodology}

To thoroughly investigate the effect of any condition of engine operation on 
engine life consumption, a thermodynamic model of the engine is created in PYTHIA [12], a gas turbine performance analysis and diagnostics software developed and validated [16] at Cranfield University, and ensure it behaves like the real engine by adapting the engine model to real engine operation conditions. LM2500+ engine is considered in this work. The engine consists of a compressor, a combustor, a HP turbine and a power turbine, with maximum power output of $30.2 \mathrm{MW}$ at $60 \mathrm{~Hz}$, sea level, $15^{\circ} \mathrm{C}$ and $60 \%$ relative humidity [13]. Figure 1 shows the engine model.

Blade thermal and stress models are developed to calculate the temperatures and the stresses required in the lifing model. Severity of engine operation is assessed in each condition by exploiting relative life analysis using the creep factor instead of the estimated remaining life. The lifing model, blade thermal and stress models, creep factor relation, and formulation of sensitivity of creep life consumption due to the various effects are presented here.

\subsection{Creep Life Model}

The Larson-Miller Parameter (LMP) method [14] is used for the creep life analysis in this work. It is expressed in Equation (1),

$$
t_{f}=10^{\left(\frac{\mathrm{LMP}}{T}-C\right)}
$$

$t_{f}$ is the time to creep failure in hrs, $T$ is the temperature of the blade material in Kelvin [K], $C$ is material constant usually of the order of 20, and LMP is the Larson-Miller Parameter. The temperature of the blade material is gotten from the blade thermal model. The LMP depends on the stress of the blade and it read from a master curve based on the stress level, where the stress is obtained from the stress model. The Larson-Miller model is quite accurate and it is widely used because of the availability of material master curve.

\subsection{Blade Thermal Model}

The blade thermal model helps in estimating the temperature of each blade at

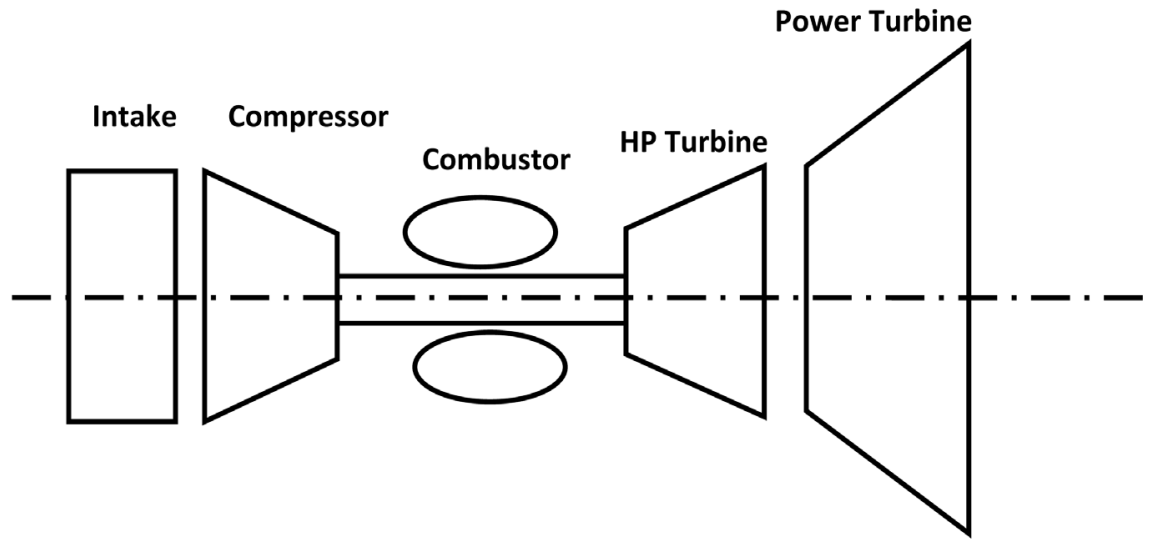

Figure 1. Configuration of the engine model. 
various sections along the span of the blade. The blade is divided into 8 equal sections along the span. Temperatures are estimated at 9 nodal points as in Figure 2 .

The blade thermal model is developed assuming convective cooling and applying radial temperature distribution. Overall energy balance of the blades is considered with hot gas traversing the blades while cooling air extracted towards the last compressor stage enters the blade through the blade root and exits at the tip. Coolant mass flow function, overall effectiveness $\varepsilon_{f f}$, and convective cooling efficiency $\eta_{c}$ [15] are employed in estimating the blade metal temperature. The maximum circumferential mean temperature of the hot gases $T_{\max }$ anywhere in the combustor exit is obtained from the radial temperature distribution factor (RTDF) relation [16], given by Equation (2),

$$
T_{\max }=T_{\text {Bur_Mean }}+\operatorname{RTDF}\left(T_{\text {Bur_Mean }}-T_{\text {Bur_In }}\right)
$$

where $T_{\text {Bur_In }}$ is the burner inlet temperature, and $T_{\text {Bur_Mean }}$ is the mean burner exit temperature. Lower values of RTDF are recommended in order to obtain uniform temperature profile [17], and this value should be less than 0.2 [18]. RTDF of 0.12 is used in this work so as to obtain uniform temperature profile. The basic assumptions made are as follows: $T_{\max }$ occurs at $62.5 \%$ distance from the blade root, minimum gas temperatures occur at the blade root and blade tip, and linear temperature distribution from the location of maximum temperature to the blade tip and blade root respectively. These assumptions are made to enable the calculation of the blade temperature at the different sections. All nodal temperatures can thus be estimated and the minimum gas temperature $T_{\min }$ is obtained as given by Equation (3),

$$
T_{\min }=\frac{9 T_{\text {Bur_Mean }}-4 T_{\max }}{5}
$$
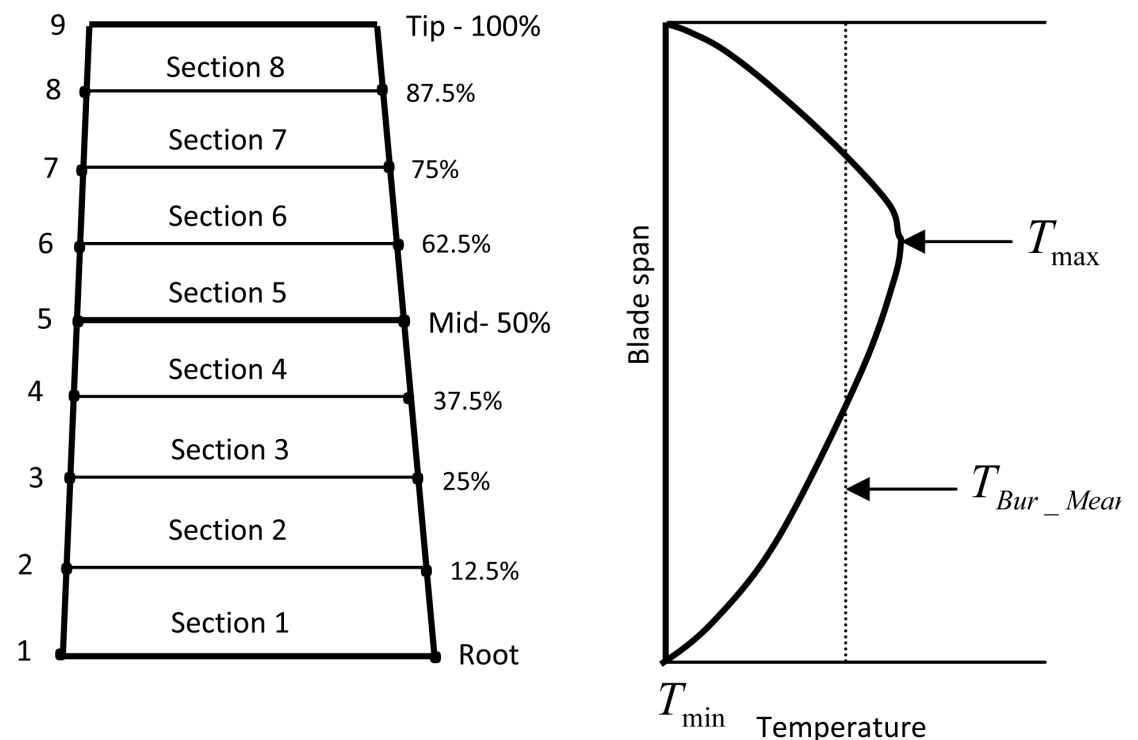

Figure 2. Blade sections and radial temperature distribution at inlet. 
The blade metal temperature at each section $T_{m, s}$ is estimated by Equation (4),

$$
T_{m, s}=T_{g, s}-\varepsilon_{f f}\left(T_{g, s}-T_{i n, s}^{c}\right)
$$

where $T_{i n, s}^{c}$ is the coolant inlet temperature at the section, and $T_{g, s}$ is the mean gas temperature of the section. Details of this model and procedure for calculations could be found in [19].

\subsection{Blade Stress Model}

In the blade stress model, the various stresses the blades encounter are estimated and the maximum stress at different locations along the span of the blade (in each of the nodes) is evaluated at three chord-wise sites which are the most likely sites for the occurrence of maximum stress: the leading edge (LE), trailing edge (TE) and furthest point at the blade suction surface. The model incorporates centrifugal stresses and bending moment stresses. The centrifugal force $F_{\text {seci }}$ at each blade section is assumed to act at the centre of gravity (CG) of the section, and it is given by Equation (5),

$$
F_{\text {seci }}=m_{\text {seci }} \times \omega^{2} \times h_{C G, i}
$$

$m_{\text {seci }}$ is the mass of the section, $\omega$ is the angular speed of the compressor shaft, (in rad/s), and $h_{C G, i}$ is the distance from the centre of gravity of the section to the axis of rotation of the turbine. The centrifugal force at node $i, F_{i}$ is given by Equation (6),

$$
F_{i}=\sum_{i=i}^{i=8} F_{\text {seci }}
$$

The centrifugal stress at each node $\sigma_{C, i}$ is given by Equation (7)

$$
\sigma_{C, i}=\frac{F_{i}}{A_{c s, i}}
$$

where $A_{c s, i}$ is the cross-sectional area of the blade at node $i$. The pressure change bending moment $M_{P, i}$ and the velocity change bending moments (in axial direction $B M_{A x, i}^{V}$, and the tangential direction $B M_{T, i}^{V}$ ) at each node are obtained and resolved in the blade direction using Equations (8) and (9).

$$
\begin{aligned}
& B M_{X X, i}=\left(B M_{P, i}+B M_{A x, i}^{V}\right) \sin \theta_{i}+B M_{T, i}^{V} \cos \theta_{i} \\
& B M_{Y Y, i}=\left(B M_{P, i}+B M_{A x, i}^{V}\right) \cos \theta_{i}-B M_{T, i}^{V} \cos \theta_{i}
\end{aligned}
$$

$B M_{X X, i}$ and $B M_{Y Y, i}$ are the axial and tangential bending moments on the blade axial and tangential directions respectively. $\theta$ is the blade stagger angle and it may vary from the blade tip to the root. The bending moment stresses at the base of each section and at the three locations are used together with the centrifugal stresses (details in [19]) to obtain the total stress and then the maximum stress at the base of each section of the blade used for the blade life calculation.

\subsection{Creep Factor at Engine Operation Point}

At any point of engine operation, the creep factor $C F_{i}[10]$ is given as the ratio 
of the creep life $C L_{i}$ estimated at that point and the reference life $C L_{\text {Ref }}$, and it is given by Equation (10),

$$
C F_{i}=\frac{C L_{i}}{C L_{\text {Ref }}}
$$

The creep factor indicates the wellness of engine operation; a value above 1 indicates favourable engine operation, a value below 1 indicates unfavourable engine operation, and 1 indicates engine operation similar to reference condition. The engine reference condition in this work is defined by engine shaft power of $30 \mathrm{MW}$, ambient temperature of $13^{\circ} \mathrm{C}$, relative humidity of $68 \%$ and ambient pressure of 1 bar.

\subsection{Life Reduction Index/Sensitivity Analysis}

In order to compare the impact of various effects on creep life, a life assessment parameter, life reduction index is introduced and applied to each effect in this work. Life reduction index is the propensity of the effect (shaft power increase or ambient temperature increase) to reduce the creep life of the engine by half. The effects do occur over a wide range of temperatures and power levels, thus the mean life reduction index will be used to cover the impact the effect has on creep life for a defined range of temperatures and shaft powers. The higher the mean life reduction index due to a given effect, the greater the impact the effect has on creep life. The mean life reduction indices are defined for the various effects here. The mean percentage reduction in creep factor $P R_{C F, m}$ at a particular power level due to unit increase in ambient temperature for the entire range of temperatures is given by Equation (11),

$$
P R_{C F, m}=\frac{\sum_{i=1}^{n-1}\left(C F_{T, i}-C F_{T, i+1}\right)}{n-1} \times 100
$$

$n$ is the number of ambient temperature levels where creep factors are estimated, $C F_{T, i}$ is the creep factor at the initial temperature value, and $C F_{T, i+1}$ is the creep factor evaluated at the next temperature value; for instance, at $-10^{\circ} \mathrm{C}$ and $-9^{\circ} \mathrm{C}$ deviation from ISA. Reduction in creep factor by $50 \%$ means the life of the blades have reduced to half the original value due to the given effect (increase in ambient temperature in this case. 50\% reduction in life is used here as a reference to compare the impacts of the different effects on creep life). The mean life reduction index MLRI due to ambient temperature increase at a particular power level is thus given by Equation (12),

$$
\text { MLRI }=\frac{P R_{C F, m}}{50}
$$

At a particular ambient temperature, the mean percentage reduction in creep factor $P R_{C F, m}$ as a result of given percentage change in shaft power is given by Equation (13), 


$$
P R_{C F, m}=\frac{\sum_{i=1}^{n-1}\left(C F_{P, i}-C F_{P, i+1}\right)}{l \times(n-1)} \times 100
$$

$n$ is the number of shaft power levels where creep factors are estimated, $l$ is the difference between successive power levels ( $l=5$ in this case), $C F_{P, i}$ is the creep factor at the initial given power level, and $C F_{P, i+1}$ is the creep factor evaluated at the next power level; for instance at $70 \%$ and $75 \%$ power levels. The mean life reduction index is calculated using Equation (12).

\section{Effect of Ambient Temperature Variation on Creep Life Consumption}

The rate of creep life consumption with ambient temperature depends on the power level of the engine. Figure 3 shows the creep factors of the first stage HP turbine blades of the engine under study at different power levels.

The creep factors decrease with increase in ambient temperature at all power levels. This is because an increase in ambient temperature leads to corresponding increase in the temperature of the hot gas the blades are exposed to. The rate of decrease in creep factors with ambient temperature at each power level is higher in the lower ambient temperature band. This is because in the lower ambient temperature band the gas is more compressible and change in ambient temperature in that range will produce more noticeable change in the properties of the gas at the combustion exit compared to the higher ambient temperature band. Also, ambient temperature has much influence on creep factor and hence creep life at lower power levels than at higher power levels; this is because at higher power levels the temperature of the gas at combustor exit is higher hence increase in ambient temperature does not lead to significant increase in the combustor exit gas.

Figure 4 shows the average percentage decrease in creep factors with ambient

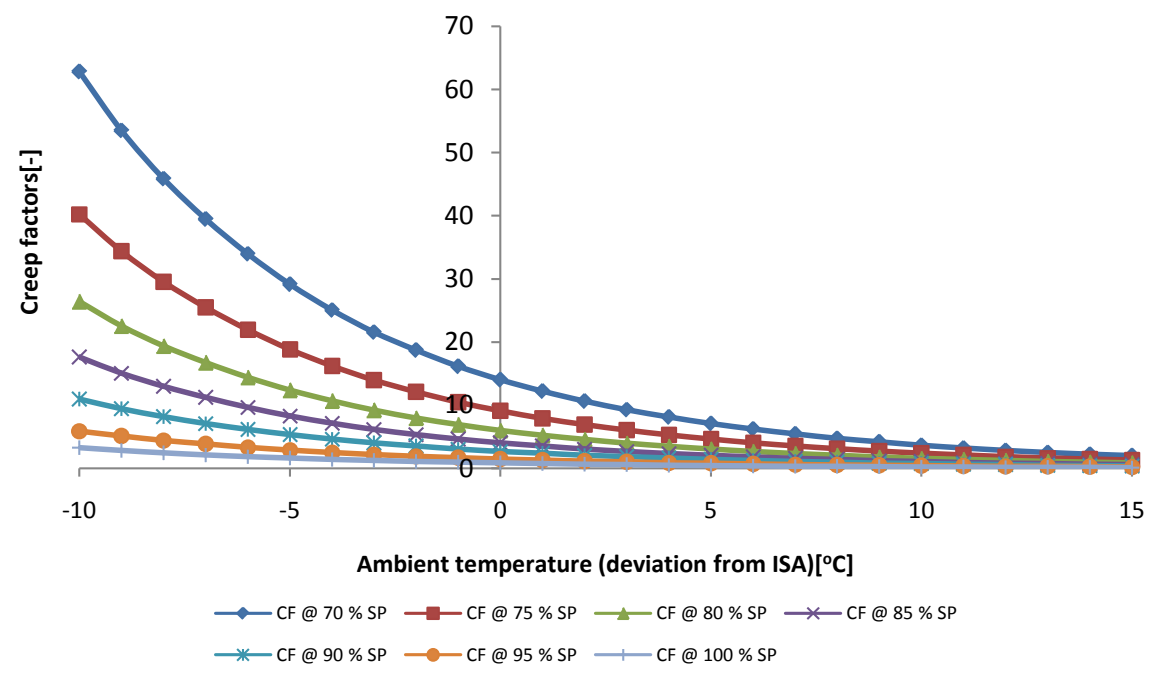

Figure 3. Variation of CF with ambient temperature. 


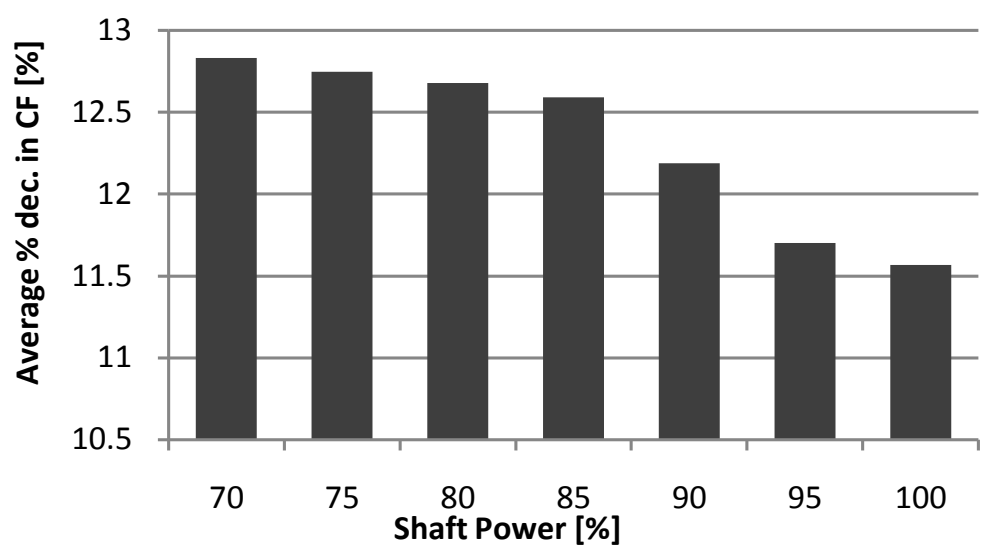

Figure 4. Percentage decrease (average) in CF with ambient temperature.

temperature at different power levels. The percentage decreases in creep factor with ambient temperature for all the range of ambient temperatures considered are used in getting the average value at each power level.

The mean life reduction index is highest at $70 \%$ power level and lowest at $100 \%$ power level, and the values are 0.2549 and 0.2313 respectively. The mean life reduction index considering all the power levels is 0.2451 . The mean percentage decrease in creep factors for all the power levels is about $12.33 \%$. Based on this value the creep life of the hot section turbine blades will be reduced by half when the ambient temperature is increased by 8.11 units. On the other hand, the creep life of the blades will increase by a factor of 2 if ambient temperature is reduced by 8.11 units.

\section{Effect of Shaft Power Variation on Creep Life Consumption}

A major consideration in industrial gas turbine operation is to consider operating at high power level or low power level. Creep life increases with decrease in shaft power and the rate of increase is higher in the higher power band (that is when power is dropped from say $100 \%$ to $95 \%$ the increase in creep life is higher compared to when power is dropped from $75 \%$ to $70 \%$ at any prevailing ambient temperature). The increase in creep factors due to drop in power from $75 \%$ power level to $70 \%$ power level and from $95 \%$ power level to $90 \%$ power level for different ambient temperatures are shown in Figure 5. At a particular power drop, the increase in creep factor is lower at higher ambient temperatures. That is, benefits in terms of increasing creep life from power drop are more pronounced at lower ambient temperatures in the higher power band. This is because at higher ambient temperatures the turbine efficiency drops hence lowering the power has less effects at this poor operation region compared to the comfortable lower ambient temperature operations.

The increase in $\mathrm{CF}$ as power drops from higher power level to lower power levels at different ambient temperatures increases initially and later drops. This is shown in Figure 6 where the mean values of the percentage increase in CF as 


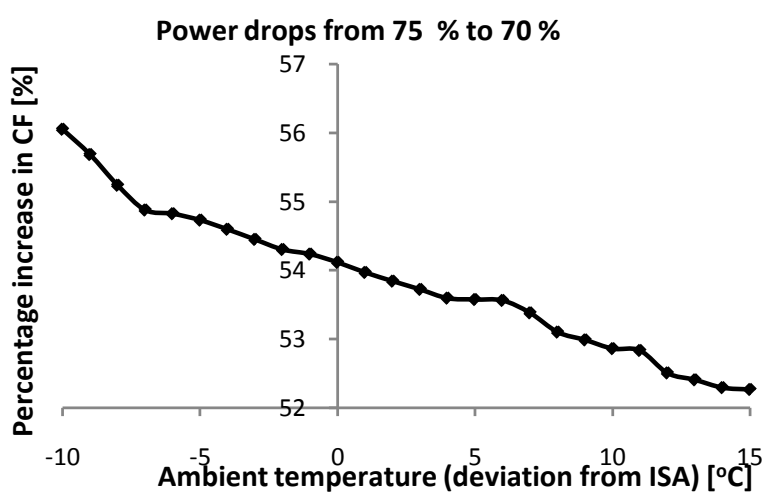

(a)

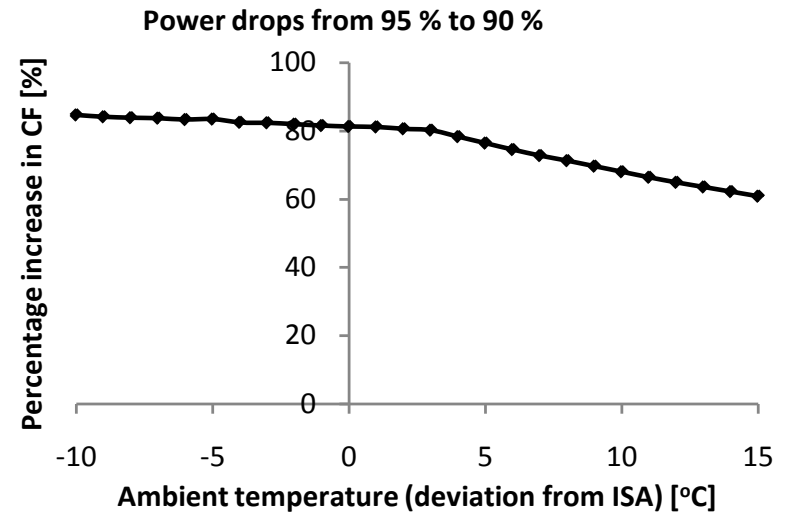

(b)

Figure 5. Percentage increase in CF with power drop.

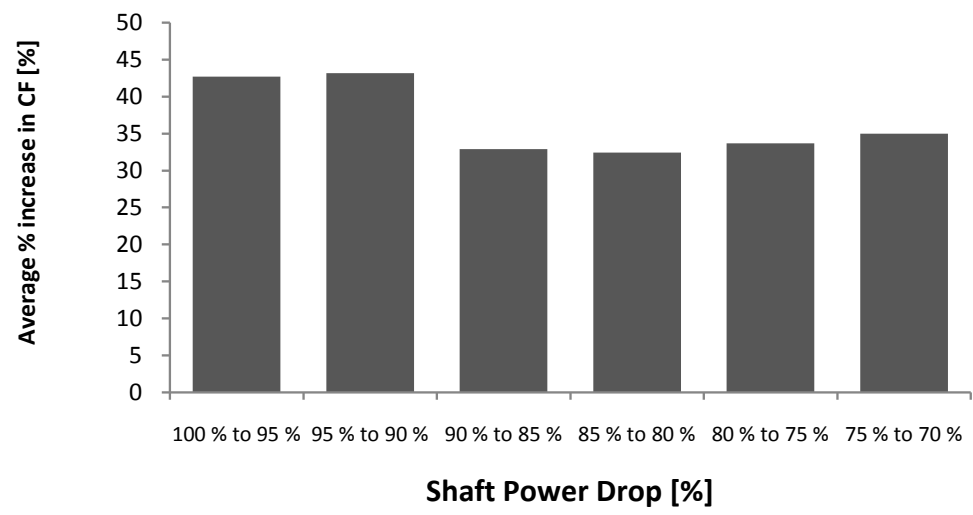

Figure 6. Average percentage increase in CF with power drop.

power drops from one level to another at different ambient temperatures are considered. Below $90 \%$ power level, the increase in CF with power drop is lower but with no proper trend.

The trend in the increase in $\mathrm{CF}$ with power drop shows that peak operation and lower power level of engine operation may be too extreme regions of engine operation and hence may not be very favourable compared to the near peak operation. Operating the engine in this case between $95 \%$ and $90 \%$ power levels if 
power demand is not too high translates to lower engine life consumption resulting from power drop. Although, the HP turbine blade life is higher at the lower power band, but the benefits in increasing creep life with power drop are higher in operating the engine around $90 \%$ to $95 \%$ power levels. The average percentage decrease in CF due to power variation reduces with ambient temperature and this is shown in Figure 7. This is because at higher ambient temperatures air becomes less compressible.

Creep factors decrease with shaft power increase in the same magnitude shaft power decrease does to creep factor increase. For $1 \%$ increase in shaft power, the lowest and the highest life reduction indices obtained at the different power levels are 0.1397 and 0.1546 respectively. The mean life reduction index considering all the power levels is 0.1466 . On the average, $1 \%$ increase in shaft power decreases creep factor by $7.33 \%$. Going by this value, the blade life will be halved when power is increased by $13.64 \%$. A unit increase in shaft power level gives a life reduction index of 0.4888 on the average.

Table 1 summarises the various effects on engine creep life consumption using the values of the mean life reduction indices.

The impact of each effect on creep life indicated by the mean life reduction indices presented in Table 1 is applicable for the ambient temperatures and shaft power levels used in analysing the creep life consumption in this work. In unit

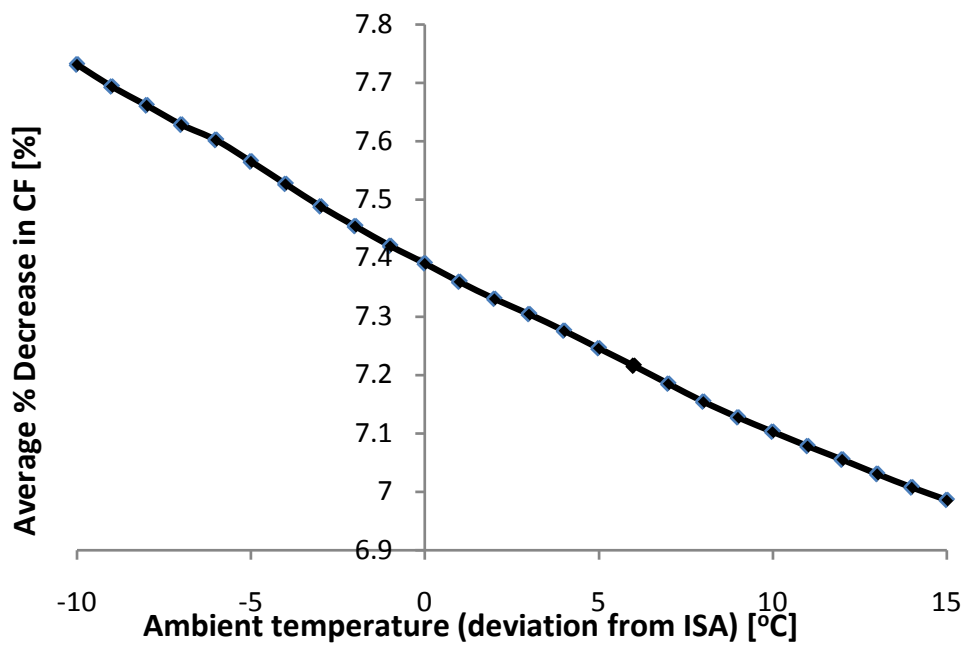

Figure 7. Average percentage decrease in $\mathrm{CF}$ with increase in shaft power.

Table 1. Summary of the impacts of various effects on creep life consumption.

\begin{tabular}{cccc}
\hline NO. & Effect & MLRI [-] & \\
\hline & & Range & Mean \\
\hline 1 & Unit increase in ambient temperature & $0.2313-0.2549$ & 0.2451 \\
2 & $1 \%$ increase in power & $0.1397-0.1546$ & 0.1466 \\
3 & Unit increase in shaft power & $0.4328-0.5762$ & 0.4888 \\
\hline
\end{tabular}


increase in ambient temperature, the lower value in the range occurs at the highest power level while the higher value occurs at the lowest power level. In shaft power increase, the lower value in the range occurs at the highest ambient temperature considered while the higher value occurs at the lowest ambient temperature. In comparing the impact of the various effects on creep life, the nature of the effect should be considered; for instance $1 \%$ increase in shaft power will not give the same life reduction index as $1 \mathrm{MW}$ increase in shaft power. Although, $1 \%$ increase in shaft power has less impact on engine creep life compared to unit increase in ambient temperature, $1 \mathrm{MW}$ increase in shaft power has far more impact on engine life than unit increase in ambient temperature.

\section{Conclusion}

The influences of shaft power variation, ambient temperature variation and engine degradation on creep life consumption have been investigated in this work. Creep factor/creep life decreases with an increase in ambient temperature. At a given power level, the rate of decrease in CF with ambient temperature is smaller at higher ambient temperatures. Creep life increases with ambient temperature drop in similar manner as ambient temperature increase does to creep life reduction. Generally, creep life reduces with an increase in shaft power but increases with decrease in shaft power. The increase in creep life as power drops from higher levels to lower levels increases initially and later drops. That is, more favourable life increase occurs near peak power operation at any ambient temperature. Knowledge of how engine creep life is consumed at different power levels and ambient temperatures as presented in this work will aid gas turbine operators in making decisions pertaining to part load operations.

\section{Acknowledgements}

The authors are grateful to the Niger Delta Development Commission (NDDC), Nigeria, and Manx Utilities (MU), Isle of Man, UK for sponsoring this research.

\section{References}

[1] Vaezi, M. and Soleymani, M. (2009) Creep Life Prediction of Inconel 738 Gas Turbine Blade. Journal of Applied Sciences, 9, 1950-1955.

https://doi.org/10.3923/jas.2009.1950.1955

[2] Marahleh, G., Kheder, A.R.I. and Hamad, H.F. (2006) Creep-Life Prediction of Service-Exposed Turbine Blades. Materials Science, 42, 476-481. https://doi.org/10.1007/s11003-006-0103-8

[3] Liu, Z., Mavris, D.N. and Volovoi, V. (2001) Creep Life Prediction of Gas Turbine Components under Varying Operating Conditions. ASME International Joint Power Generation Conference, 4-7 June 2001, New Orleans, 1-7.

[4] McLean, D. (1966) The Physics of High Temperature Creep in Metals. Reports on Progress in Physics, 29, 1-33. https://doi.org/10.1088/0034-4885/29/1/301

[5] Hanson, M.P. (1972) Effect of Temperature on Tensile and Creep Characteristics of PRD49 Fibre/Epoxy Composites. NASA, Washington DC, 1-13. 
[6] Terada, Y. and Sato, T. (2010) Assessment of Creep Rupture Life of Heat Resistant Mg-Al-Ca Alloys. Journal of Alloys and Compounds, 504, 261-264. https://doi.org/10.1016/j.jallcom.2010.05.108

[7] Kong, Z. and Li, S. (2013) Effects of Temperature and Stress on the Creep Behavior of a Ni 3 Al Base Single Crystal Alloy. Progress in Natural Science: Materials International, 23, 205-210. https://doi.org/10.1016/j.pnsc.2013.03.005

[8] Eshati, S., Abu, A., Laskaridis, P. and Haslam, A. (2011) Investigation into the Effects of Operating Conditions and Design Parameters on the Creep Life of High Pressure Turbine Blades in a Stationary Gas Turbine Engine. Mechanics and Mechanical Engineering, 15, 237-247.

[9] Abdul Ghafir, M.F., Li, Y.G., Wang, L. and Zhang, W. (2011) Impact Analysis of Aero-engine Performance Parameter Variation on Hot Section's Creep Life Using Creep Factor Approach. AIAA, 16, 1-12.

[10] Abdul Ghafir, M.F., Li, Y.G., Singh, R., Huang, K. and Feng, X. (2010) Impact of Operating and Health Conditions on Aero Gas Turbine Hot Section Creep Life Using a Creep Factor Approach. Proceedings of ASME Turbo Expo 2010: Power for Land, Sea and Air, Glasgow, 14-16 June 2010, 1-13.

[11] Robinson, S.L. (1952) Effect of Temperature Variation on the Long Time Rupture Strength of Steels. Transactions of ASME, 74, 777-780.

[12] Li, Y.G. and Singh, R. (2005) An Advanced Gas Turbine Gas Path Diagnostic System-PYTHIA. The XVII International Symposium on Air Breathing Engines, Munich, 4-9 September 2005, 1-12.

[13] GE-Marine (2014) LM2500+ Marine Gas Turbine. https://www.geaviation.com/sites/default/files/datasheet-lm2500plus.pdf

[14] Larson, F.R. and Miller, J. (1952) Time-Temperature Relationship for Rupture and Creep Stresses. Transactions of ASME, 74, 765-771.

[15] Rubini, P.A. (2014) Turbine Blade Cooling. MSc Lecture Notes, Cranfield University, Cranfield, Bedfordshire.

[16] Lefebvre, A.H. (1999) Gas Turbine Combustion. 2nd Edition, Taylor and Francis, Philadelphia.

[17] Holland, M.J. and Thake, T.F. (1980) Rotor Blade Cooling in High Pressure Turbines. Journal of Aircraft, 17, 412-418. https://doi.org/10.2514/3.44668

[18] Walsh, P. and Fletcher, P. (2004) Gas Turbine Performance. 2nd Edition, Blackwell Publishing Company, Oxford. https://doi.org/10.1002/9780470774533

[19] Saturday, E.G., Li, Y.G., Ogiriki, E.A. and Newby, M.A. (2017) Creep-Life Usage Analysis and Tracking for Industrial Gas Turbines. Journal of Propulsion and Power, 33, 1305-1314. https://doi.org/10.2514/1.B35912 